\title{
Metodologia de ensino e percepção da qualidade de vida e estresse em graduandos de enfermagem no nordeste brasileiro
}

\author{
Teaching methodology and perception of quality of life and stress in nursing students in \\ northeastern Brazil
}

Metodología de la enseñanza y percepción de la calidad de vida y el estrés en estudiantes de enfermería del noreste de Brasil

\section{Resumo}

Objetivo: avaliar a influência do método de ensino aprendizagem na percepção de qualidade de vida (QV) e estresse dos acadêmicos de enfermagem de uma universidade federal brasileira. Método: estudo transversal realizado em dois campi uma universidade pública, dados coletados por meio de um questionário contendo itens para caracterização socioeconômica, acadêmica e hábitos de vida, o WHOQOL-bref e a PSS-10, para avaliação da QV e da percepção de estresse, respectivamente. A análise estatística foi realizada por meio dos testes Exato de Fisher, Qui-quadrado, teste T, ANOVA e correlação de Pearson. Os resultados com $\mathrm{p}<0,05$ foram considerados significativos Resultados: a amostra foi composta por 113 estudantes do campus ativo e 130 do campus tradicional, predominantemente do sexo feminino $(194 ; 79,8 \%)$, não brancos $(183 ; 75,3 \%)$, com uma média de idade de 22,52 $\pm 4,67$ anos, residentes na zona urbana $(220 ; 90,5 \%)$, que viviam com a família (126;51,9\%), não exerciam atividade laboral remunerada $(203 ; 83,5 \%)$

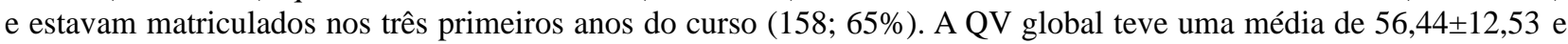

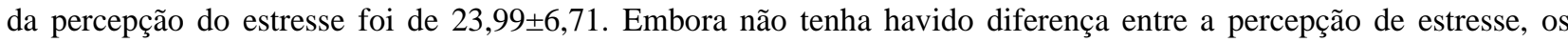
estudantes do método ativo apresentaram melhores escores de QV global e nos domínios psicológico, social e ambiental $(\mathrm{p}<0,05)$. Conclusão: os estudantes do método ativo apresentaram melhores resultados na avaliação da QV, porém não houve diferenças significativas quanto à percepção do estresse entre os grupos avaliados.

Palavras-chave: Aprendizagem ativa; Ensino; Estudantes de enfermagem; Estresse psicológico; Qualidade de vida.

\begin{abstract}
Objective: to evaluate the influence of the teaching-learning method on the perception of quality of life (QL) and stress among nursing students at a Brazilian federal university. Method: cross-sectional study carried out on two campuses of a public university, data collected through a questionnaire containing items for socioeconomic, academic and lifestyle habits, the WHOQOL-bref and the PSS-10, to assess QoL and perception of stress, respectively. Statistical analysis was performed using Fisher's Exact, Chi-square, T-test, ANOVA and Pearson's correlation tests. Results with $\mathrm{p}<0.05$ were considered significant. Results: the sample consisted of 113 students from the active campus and 130 from the traditional campus, predominantly female (194;79.8\%), non-white $(183 ; 75.3 \%)$, with a mean age of $22.52 \pm 4.67$ years, residing in the urban area $(220.90 .5 \%)$, who lived with their family $(126 ; 51.9 \%)$, did not exercise paid work $(203 ; 83.5 \%)$ and were enrolled in the first three years of the course $(158 ; 65 \%)$. The global
\end{abstract}


QoL had an average of $56.44 \pm 12.53$ and the perception of stress was $23.99 \pm 6.71$. Although there was no difference between the perception of stress, the active method students had better global QoL scores and in the psychological, social, and environmental domains $(\mathrm{p}<0.05)$. Conclusion: the active method students showed better results in the assessment of QoL, but there were no significant differences in the perception of stress between the evaluated groups.

Keywords: Problem-based learning; Teaching; Students, Nursing; Stress, Psychological; Quality of life.

\section{Resumen}

Objetivo: evaluar la influencia del método de enseñanza-aprendizaje en la percepción de la calidad de vida (CV) y el estrés en estudiantes de enfermería de una universidad federal brasileña. Método: estudio transversal realizado en dos campus de una universidad pública, datos recolectados a través de un cuestionario que contiene ítems de hábitos socioeconómicos, académicos y de estilo de vida, el WHOQOL-bref y el PSS-10, para evaluar la CV y la percepción de estrés, respectivamente. . El análisis estadístico se realizó utilizando Exacto de Fisher, Chi-cuadrado, prueba T, ANOVA y pruebas de correlación de Pearson. Se consideraron significativos los resultados con $\mathrm{p}<0.05$. Resultados: la muestra estuvo conformada por 113 estudiantes del campus activo y 130 del campus tradicional, predominantemente mujeres $(194 ; 79,8 \%)$, no blancos $(183 ; 75,3 \%)$, con una media edad de 22,52 $\pm 4,67$ años, residentes en el área urbana $(220 ; 90,5 \%)$, que vivían con su familia $(126 ; 51,9 \%)$, no ejercían trabajo remunerado $(203 ; 83,5 \%)$ y estaban matriculados en los primeros tres años del curso $(158 ; 65 \%)$. La CV global tuvo una media de $56,44 \pm 12,53$ y la percepción de estrés fue de $23,99 \pm 6,71$. Aunque no hubo diferencia entre la percepción del estrés, los estudiantes del método activo tuvieron mejores puntuaciones globales de CV y en los dominios psicológico, social y ambiental $(\mathrm{p}<0,05)$. Conclusión: los estudiantes del método activo mostraron mejores resultados en la evaluación de la CV, pero no hubo diferencias significativas en la percepción de estrés entre los grupos evaluados.

Palabras clave: Aprendizaje activo; Enseñanza; Estudiantes de enfermería; Estrés psicológico; Calidad de vida.

\section{Introdução}

Impulsionadas pela crescente globalização e influenciadas por fatores socioeconômicos, mudanças nos programas de formação do enfermeiro são exigidas em todo mundo (Jeppesen, Christiansen \& Frederiksen, 2017; WHO, 2009). A fim de alinhar o avanço profissional às tendências da educação mundial e atender às expectativas dos sistemas de prestação de cuidados de saúde em constante mudança, observa-se no Brasil uma tendência de implementação de métodos inovadores de ensino que valorizam o saber livre e ativo do graduando na construção do conhecimento (Pinto, Marin, Tonhom \& Ferreira, 2016).

Apesar disso, o ensino tradicional, no qual o professor é o detentor do conhecimento, ainda é prevalente. Nessa abordagem, o docente atua, geralmente, com aulas expositivas, o que cria um fluxo unilateral de comunicação e leva a uma dificuldade no desenvolvimento do pensamento crítico por parte do estudante (Magnavita \& Chiorri, 2018; Quadro \& Chiorri, 2018).

A ferramenta mais utilizada no processo ensino-aprendizagem ativo em enfermagem, a aprendizagem baseada em problemas (Problem Based Learning- PBL) (Pinto et al., 2016), incentiva o raciocínio clínico, a investigação do conhecimento e as habilidades adquiridas ao longo da vida, o que torna o aprendizado mais relevante e aplicável na preparação dos alunos para suas carreiras (Wardley, Applegate, Almaleki, \& Van Rhee, 2016).

O desempenho a longo prazo dos alunos de enfermagem submetidas a PBL mostrou-se superior aos do método convencional (Salari, Roozbehi, Zarifi, \& Tarmizi, 2018). No entanto, um estudo revelou que alunos que seguiam a metodologia PBL mostraram-se mais estressados durante a maior parte do programa de graduação, em comparação aos alunos que utilizavam o método de aprendizagem baseado em aulas expositivas (Wardley et al., 2016).

Adquirir conhecimentos e desenvolver habilidades e atitudes é importante na formação do enfermeiro. Porém, devido às demandas do processo formativo, alguns fatores estressantes podem interferir na qualidade de vida $(\mathrm{QV})$ dos estudantes de enfermagem, como pouca ou nenhuma oportunidade de lazer, estresse, sobrecarga de atividades curriculares e estágios, período integral do curso, falta de tempo para atividades extracurriculares, percepção de conhecimento teórico-prático insuficiente (Gouveia et al., 2017; Hirsch, Luiz, Barlem \& Almeida, 2018), além da prática clínica e ao medo de causar danos aos pacientes ou a si mesmos (Suárez-García et al., 2018). 
Nesse contexto, faz-se necessário investigar a QV e o estresse entre estudantes de enfermagem. A QV é conceituada como a percepção do indivíduo de sua posição na vida no contexto da cultura e sistemas de valores, nos quais ele vive e em relação a seus objetivos, expectativas, padrões e preocupações (WHO, 1997). Já o estresse pode ser definido como uma resposta inespecífica do organismo a estímulos nocivos que pode ser observada e mensurada (Selye, 1951). Portanto, esse estudo teve por objetivo avaliar se o método de ensino-aprendizagem influencia na percepção de QV e estresse dos acadêmicos de enfermagem de uma universidade federal brasileira.

\section{Metodologia}

Trata-se de um estudo observacional de corte transversal com abordagem descritiva e quantitativa realizado em dois campi de uma Universidade Federal do Nordeste que apresentam abordagens pedagógicas distintas para o ensino da enfermagem: metodologia tradicional e ativa.

O estudo obedeceu aos preceitos éticos da Resolução no 466/2012 do Conselho Nacional de Saúde. A coleta de dados foi realizada de outubro de 2017 a fevereiro de 2018, após aprovação pelo Comitê de Ética em Pesquisa (CAAE ${ }^{\circ}$ 61993616.3.0000.5546 e parecer $n^{\circ}$ 1.917.559). Foram incluídos na pesquisa os alunos da graduação em enfermagem de todas as séries com matrícula ativa durante o período da coleta e presentes nas datas de coleta de dados.

Amostra não paramétrica e não probabilística de conveniência numa população de 193 e 332 graduandos de enfermagem com matrícula ativa dos campi ativo e tradicional, respectivamente, de uma universidade. Foi determinada após a realização de um teste piloto com dez alunos em cada campus que resultou numa amostra mínima de 103 alunos por campus.

Os pesquisadores compareceram aos campi diariamente e solicitavam autorização dos docentes para usar um curto período no final de suas aulas. Em seguida, esclareciam sobre o objetivo da pesquisa, solicitado o consentimento dos voluntários, por meio de anuência verbal e escrita com assinatura do termo de consentimento livre e esclarecido (TCLE).

Os instrumentos de coleta de dados foram distribuídos em envelopes sem identificação para garantir a confidencialidade dos dados. Um questionário foi elaborado pelos pesquisadores para caracterização do perfil sociodemográfico, econômico e acadêmico. Para avaliar a presença de estresse entre os graduandos, foi utilizada a Perceived Stress Scale-10 (PSS-10), instrumento de relato pessoal, capaz de mensurar, de forma global, até que ponto os acontecimentos de vida são percebidos como indutores e contribuintes do estresse (Cohen, Kamarck \& Mermelstein, 1983). Essa escala é composta por 10 itens (seis positivos e quatro negativos) com opções de resposta que variam de zero a quatro ( $0=$ nunca; $1=$ quase nunca; $2=$ às vezes; $3=$ quase sempre; $4=$ sempre) (Cohen \& Williamson, 1988).

Para coleta dos dados quantitativos acerca da QV, foi utilizado o World Health Organization Quality of Life Group (WHOQOL-bref), elaborado pela Organização Mundial de Saúde (OMS). Esse instrumento é composto por dois itens da QV Geral e Saúde Geral e 24 itens distribuídos em quatro domínios de QV: físico, psicológico, relações sociais e meio ambiente (WHO, 1997). No tocante a avaliação da QV dos universitários, os escores obtidos foram transformados em escala de 0 a 100, sendo considerado os valores próximos a zero (0) como insatisfação e a cem (100) satisfação a respeito da QV.

Foi realizada análise descritiva dos dados, na qual as variáveis qualitativas foram expressas por meio de frequências absolutas e relativas e as variáveis numéricas representadas por meio de medidas de tendência central e dispersão (média \pm desvio padrão). Os testes Qui-quadrado de Pearson e Exato de Fisher foram usados para avaliar a significância estatística entre os dados categóricos por campus.

Para avaliar as correlações entre QV e seus domínios (WHOQOL-bref) e estresse (PSS) foi utilizado a correlação linear de Pearson. A comparação dos escores de QV e PSS entre os estudantes dos campi e demais variáveis foi analisada por meio do teste $\mathrm{T}$ de Student independente e ANOVA. Foi estabelecida significância de $5 \%(\mathrm{p}<0,05)$ em todas as análises realizadas. 


\section{Resultados}

Participaram do estudo um total de 243 estudantes de enfermagem, desses 113 alunos eram do campus ativo e 130 do campus tradicional. As características sociodemográficas dos participantes por campus estão resumidas na Tabela 1. A maior proporção dos participantes foi do sexo feminino, com uma média de idade de 22,52 $\pm 4,67$ anos (17 a 52 anos), sem companheiros e residentes na área urbana.

Tabela 1. Características sociodemográficas, acadêmicas e hábitos de vida dos graduandos de enfermagem.

\begin{tabular}{|c|c|c|c|}
\hline Variáveis & Campus Ativo & $\begin{array}{c}\text { Campus } \\
\text { Tradicional }\end{array}$ & $p$ \\
\hline Idade (média $\pm \mathrm{DP})$ & $22,01 \pm 3,97$ & $22,93 \pm 5,14$ & $0,132 * *$ \\
\hline Sexo n $(\%)$ & $105(100,0)$ & $134(100)$ & \multirow{3}{*}{0,112} \\
\hline Feminino & $90(85,7)$ & $104(77,0)$ & \\
\hline Masculino & $15(14,3)$ & $30(22,0)$ & \\
\hline Zona de residência n $(\%)$ & $108(100,0)$ & $135(100,0)$ & \multirow{3}{*}{$<0,001 *$} \\
\hline Urbana & $89(82,4)$ & $131(97,0)$ & \\
\hline Rural & $19(17,6)$ & $4(3,0)$ & \\
\hline Cor da pele n $(\%)$ & $108(100,0)$ & $134(100,0)$ & \multirow{3}{*}{0,689} \\
\hline Branco & $25(23,1)$ & $34(25,4)$ & \\
\hline Não branco & $83(76,9)$ & $100(74,6)$ & \\
\hline Estado civil n (\%) & $108(100)$ & $135(100,0)$ & \multirow{3}{*}{0,559} \\
\hline Com companheiro & $11(10,2)$ & $17(12,6,0)$ & \\
\hline Sem companheiro & $97(89,8)$ & $118(87,4)$ & \\
\hline Reside n $(\%)$ & $103(100)$ & $125(100,0)$ & \multirow{4}{*}{0,041} \\
\hline Sozinho & $7(6,5)$ & $11(8,1)$ & \\
\hline Em república & $67(62,0)$ & $17(12,6)$ & \\
\hline Com a família & $29(26,9)$ & $97(71,9)$ & \\
\hline Renda familiar n (\%) & $108(100)$ & $130(100,0)$ & \multirow{6}{*}{0,001} \\
\hline Sem renda & $25(24,0)$ & $7(5,4)$ & \\
\hline Até $1 \mathrm{SM}$ & $19(18,3)$ & $24(18,5)$ & \\
\hline 1 a 3 SM & $33(31,7)$ & $48(36,9)$ & \\
\hline 3 a 6 SM & $16(15,4)$ & $36(27,7)$ & \\
\hline Mais de $6 \mathrm{SM}$ & $11(10,6)$ & $15(11,5)$ & \\
\hline Trabalha n (\%) & $108(100,0)$ & $127(100,0)$ & \multirow{3}{*}{0,068} \\
\hline Sim & $9(8,5)$ & $21(16,5)$ & \\
\hline Não & $97(81,5)$ & $106(83,5)$ & \\
\hline Ano n $(\%)$ & $108(100,0)$ & $135(100,0)$ & \multirow{6}{*}{0,324} \\
\hline Primeiro ano & $28(25,9)$ & $23(17,0)$ & \\
\hline Segundo ano & $27(25,0)$ & $32(23,7)$ & \\
\hline Terceiro ano & $22(20,4)$ & $26(19,3)$ & \\
\hline Quarto ano & $23(21,3)$ & $38(28,1)$ & \\
\hline Quinto ano & $8(7,4)$ & $16(11,9)$ & \\
\hline Pesquisa n $(\%)$ & $107(100,0)$ & $135(100,0)$ & \multirow{3}{*}{0,429} \\
\hline Sim & $57(53,3)$ & $65(48,1)$ & \\
\hline Não & $50(46,7)$ & $70(51,9)$ & \\
\hline Monitoria n (\%) & $108(100,0)$ & $135(100)$ & \multirow{3}{*}{0,233} \\
\hline Sim & $21(19,4)$ & $35(25,9)$ & \\
\hline Não & $87(80,6)$ & $100(74,1)$ & \\
\hline Extensão n (\%) & $108(100,0)$ & $135(100)$ & \multirow{3}{*}{0,160} \\
\hline Sim & $61(56,5)$ & $64(47,4)$ & \\
\hline Não & $47(43,5)$ & $71(52,6)$ & \\
\hline Refeição balanceada n (\%) & $108(100,0)$ & $135(100)$ & \multirow{3}{*}{0,497} \\
\hline Sim & $92(85,2)$ & $119(88,1)$ & \\
\hline Não & $16(14,8)$ & $16(11,9)$ & \\
\hline Tabagismo n (\%) & $107(100,0)$ & $135(100,0)$ & \multirow{3}{*}{$0,137 *$} \\
\hline Sim & $1(0,9)$ & $6(4,4)$ & \\
\hline Não & $106(99,1)$ & $129(95,6)$ & \\
\hline Etilismo n (\%) & $107(100,0)$ & $134(100,0)$ & \multirow{3}{*}{0,967} \\
\hline Sim & $33(30,8)$ & $41(30,6)$ & \\
\hline Não & $74(69,2)$ & $93(69,4)$ & \\
\hline Dorme $7 / 8$ horas $\mathrm{n}(\%)$ & $108(100,0)$ & $135(100,0)$ & \multirow{3}{*}{0,733} \\
\hline Sim & $26(24,1)$ & $30(22,2)$ & \\
\hline Não & $82(75,9)$ & $105(77,8)$ & \\
\hline Atividade Social n (\%) & $107(100)$ & $129(100,0)$ & \multirow{3}{*}{0,695} \\
\hline Sim & $62(57,9)$ & $78(60,5)$ & \\
\hline Não & $45(42,1)$ & $51(39,5)$ & \\
\hline
\end{tabular}

*Teste Exato de Fisher. **Teste T-student. Fonte: Autores.

Os estudantes não trabalhavam e não recebiam auxílio da universidade. A renda familiar concentrou-se entre 1 e 3 
salários mínimos, seguido por nenhuma renda no campus ativo e 3 a 6 salários mínimos no campus tradicional. Quanto às características acadêmicas, a maioria dos participantes do método ativo estavam no segundo ano, enquanto os estudantes do método tradicional estavam no quarto ano. Em geral, os alunos estavam ou já estiveram inseridos em projetos de iniciação científica e em projetos de extensão e poucos em monitoria.

A respeito dos hábitos de vida, a maioria dos estudantes referiram pelo menos uma refeição balanceada por dia e desempenhavam atividades sociais regularmente. A proporção de tabagistas foi mínima, assim como dos que referiram consumir bebidas alcoólicas. Houve diferença significativa na comparação por campus entre as variáveis zona de residência, renda familiar e com quem reside $(\mathrm{p}<0,05)$.

Em geral, a maior QV foi registrada no domínio social $(62,62 \pm 18,76)$, seguido pelos domínios psicológico $(55,68 \pm 15,57)$ e físico $(55,72 \pm 14,91)$, e a menor no domínio ambiental $(51,75 \pm 13,79)$. A QV global teve uma média de $56,44 \pm 12,53$. A média de percepção do estresse foi de $23,99 \pm 6,71$.

Nesse estudo, houve correlações significativas e decrescentes entre o estresse e a QV geral e todos os domínios de QV (Figura 1). Na QV geral e nos domínios físico e psicológico a correlação negativa foi moderada e nos domínios social e ambiental, fraca.

Figura 1. Gráficos da correlação linear de Pearson entre PSS e as facetas do WHOQOL-bref.
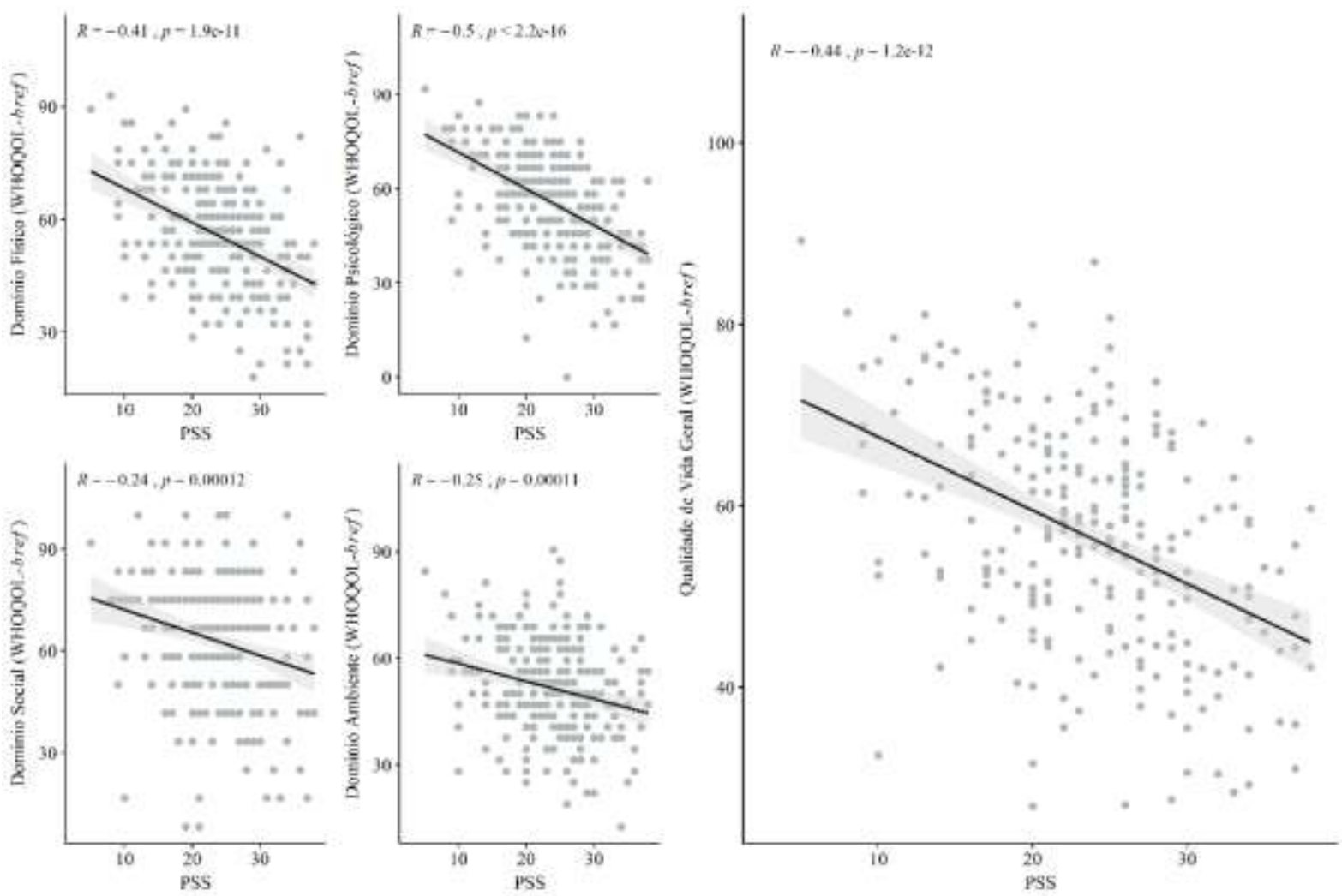

Fonte: Autores.

Alunos do método ativo obtiveram melhores escores de QV global e nos domínios psicológico, social e ambiental $(\mathrm{p}<0,05)$ em relação aos alunos do método tradicional. Estudantes do sexo masculino obtiveram maior escore no domínio físico. Não houve diferença significativa nas correlações entre QV e as variáveis cor da pele, zona de residência e estado civil (Tabela 2). 
Research, Society and Development, v. 10, n. 7, e30810716626, 2021

(CC BY 4.0) | ISSN 2525-3409 | DOI: http://dx.doi.org/10.33448/rsd-v10i7.16626

Tabela 2. Associação entre qualidade de vida, estresse percebido, características sociodemográficas, acadêmicas e hábitos de vida.

\begin{tabular}{|c|c|c|c|c|c|c|c|c|c|c|c|c|}
\hline \multirow{2}{*}{ Variáveis } & \multicolumn{2}{|c|}{ Domínio Físico } & \multicolumn{2}{|c|}{ Domínio Psicológico } & \multicolumn{2}{|c|}{ Domínio Social } & \multicolumn{2}{|c|}{ Domínio Ambiental } & \multicolumn{2}{|c|}{ QV global } & \multicolumn{2}{|l|}{ PSS } \\
\hline & Média $\pm D P$ & $p$ & Média $\pm \mathrm{DP}$ & $p$ & Média \pm DP & $p$ & Média \pm DP & $p$ & Média $\pm \mathrm{DP}$ & $p$ & Média $\pm \mathrm{DP}$ & $p$ \\
\hline \multicolumn{13}{|l|}{ Campus } \\
\hline $\begin{array}{l}\text { Tradicional } \\
\text { Ativo }\end{array}$ & $\begin{array}{l}54,97 \pm 14,76 \\
57,07 \pm 14,58\end{array}$ & 0,268 & $\begin{array}{l}52,80 \pm 15,69 \\
59,49 \pm 14,86\end{array}$ & 0,001 & $\begin{array}{l}60,49 \pm 16,69 \\
65,97 \pm 20,06\end{array}$ & 0,021 & $\begin{array}{l}49,39 \pm 13,31 \\
54,52 \pm 13,57\end{array}$ & 0,003 & $\begin{array}{l}54,41 \pm 11,67 \\
59,26 \pm 12,96\end{array}$ & 0,002 & $\begin{array}{l}23,78 \pm 6,78 \\
23,43 \pm 6,63\end{array}$ & 0,687 \\
\hline \multicolumn{13}{|l|}{ Sexo } \\
\hline $\begin{array}{l}\text { Feminino } \\
\text { Masculino }\end{array}$ & $\begin{array}{l}54,84 \pm 14,12 \\
60,39 \pm 16,65\end{array}$ & 0,023 & $\begin{array}{l}55,56 \pm 14,92 \\
56,94 \pm 19,15\end{array}$ & 0,597 & $\begin{array}{l}62,67 \pm 18,33 \\
63,70 \pm 19,55\end{array}$ & 0,738 & $\begin{array}{l}51,91 \pm 13,17 \\
50,56 \pm 16,12\end{array}$ & 0,554 & $\begin{array}{l}56,24 \pm 12,06 \\
57,90 \pm 14,52\end{array}$ & 0,424 & $\begin{array}{l}23,98 \pm 6,72 \\
22,31 \pm 6,76\end{array}$ & 0,134 \\
\hline \multicolumn{13}{|l|}{ Cor da pele } \\
\hline $\begin{array}{l}\text { Branco } \\
\text { Não branco }\end{array}$ & $\begin{array}{l}58,05 \pm 13,56 \\
55,17 \pm 15,03\end{array}$ & 0,191 & $\begin{array}{l}56,14 \pm 14,10 \\
55,67 \pm 16,20\end{array}$ & 0,842 & $\begin{array}{l}62,57 \pm 18,13 \\
63,11 \pm 18,59\end{array}$ & 0,847 & $\begin{array}{l}53,56 \pm 12,39 \\
51,14 \pm 14,00\end{array}$ & 0,236 & $\begin{array}{l}57,58 \pm 11,74 \\
56,26 \pm 12,74\end{array}$ & 0,485 & $\begin{array}{l}24,25 \pm 7,56 \\
23,44 \pm 6,43\end{array}$ & 0,421 \\
\hline \multicolumn{13}{|l|}{ Zona de residência } \\
\hline $\begin{array}{l}\text { Urbana } \\
\text { Rural }\end{array}$ & $\begin{array}{l}56,21 \pm 14,55 \\
52,95 \pm 15,98\end{array}$ & 0,312 & $\begin{array}{l}55,87 \pm 15,77 \\
54,88 \pm 14,79\end{array}$ & 0,775 & $\begin{array}{l}62,99 \pm 17,82 \\
62,31 \pm 23,95\end{array}$ & 0,867 & $\begin{array}{l}51,81 \pm 13,92 \\
50,28 \pm 10,82\end{array}$ & 0,610 & $\begin{array}{l}56,72 \pm 12,49 \\
55,10 \pm 12,50\end{array}$ & 0,554 & $\begin{array}{l}23,54 \pm 6,70 \\
24,43 \pm 6,82\end{array}$ & 0,546 \\
\hline \multicolumn{13}{|l|}{ Estado civil } \\
\hline $\begin{array}{l}\text { Com companheiro } \\
\text { Sem companheiro }\end{array}$ & $\begin{array}{l}56,24 \pm 13,76 \\
55,86 \pm 14,84\end{array}$ & 0,897 & $\begin{array}{l}59,22 \pm 14,72 \\
55,32 \pm 15,75\end{array}$ & 0,216 & $\begin{array}{l}62,79 \pm 22,85 \\
62,94 \pm 17,83\end{array}$ & 0,967 & $\begin{array}{l}54,25 \pm 12,67 \\
51,33 \pm 13,75\end{array}$ & 0,288 & $\begin{array}{l}58,12 \pm 13,12 \\
56,36 \pm 12,40\end{array}$ & 0,484 & $\begin{array}{l}22,78 \pm 5,88 \\
23,74 \pm 6,81\end{array}$ & 0,480 \\
\hline \multicolumn{13}{|l|}{ Trabalha } \\
\hline $\begin{array}{l}\text { Sim } \\
\text { Não }\end{array}$ & $\begin{array}{l}58,32 \pm 14,35 \\
55,12 \pm 14,67\end{array}$ & 0,264 & $\begin{array}{l}55,42 \pm 15,24 \\
55,45 \pm 15,85\end{array}$ & 0,991 & $\begin{array}{l}55,00 \pm 20,94 \\
63,71 \pm 18,01\end{array}$ & 0,016 & $\begin{array}{l}52,41 \pm 10,74) \\
51,33 \pm 13,84)\end{array}$ & 0,684 & $\begin{array}{l}55,29 \pm 11,50) \\
56,40 \pm 12,60)\end{array}$ & 0,649 & $\begin{array}{l}23,13 \pm 5,94) \\
23,89 \pm 6,78)\end{array}$ & 0,560 \\
\hline \multicolumn{13}{|l|}{ Monitoria } \\
\hline $\begin{array}{l}\text { Sim } \\
\text { Não }\end{array}$ & $\begin{array}{l}57,34 \pm 15,42 \\
55,47 \pm 14,48\end{array}$ & 0,407 & $\begin{array}{l}56,02 \pm 16,98 \\
55,70 \pm 15,28\end{array}$ & 0,893 & $\begin{array}{l}64,73 \pm 18,93 \\
62,38 \pm 18,29\end{array}$ & 0,405 & $\begin{array}{l}50,73 \pm 14,46) \\
51,95 \pm 13,41)\end{array}$ & 0,559 & $\begin{array}{l}57,20 \pm 13,63) \\
56,37 \pm 12,14)\end{array}$ & 0,665 & $\begin{array}{l}23,23 \pm 7,16) \\
23,74 \pm 6,57)\end{array}$ & 0,614 \\
\hline \multicolumn{13}{|l|}{ Extensão } \\
\hline $\begin{array}{l}\text { Sim } \\
\text { Não }\end{array}$ & $\begin{array}{l}56,71 \pm 15,49 \\
55,05 \pm 13,81\end{array}$ & 0,380 & $\begin{array}{l}57,33 \pm 16,03 \\
54,13 \pm 15,14\end{array}$ & 0,112 & $\begin{array}{l}65,40 \pm 17,58 \\
60,31 \pm 19,01\end{array}$ & 0,031 & $\begin{array}{l}52,91 \pm 14,23) \\
50,35 \pm 12,92)\end{array}$ & 0,145 & $\begin{array}{l}58,08 \pm 13,19) \\
54,96 \pm 11,50)\end{array}$ & 0,051 & $\begin{array}{l}23,62 \pm 6,99) \\
23,63 \pm 6,42)\end{array}$ & 0,989 \\
\hline \multicolumn{13}{|l|}{ Pesquisa } \\
\hline $\begin{array}{l}\text { Sim } \\
\text { Não }\end{array}$ & $\begin{array}{l}56,49 \pm 14,94 \\
55,41 \pm 14,49 \\
\end{array}$ & 0,568 & $\begin{array}{l}57,65 \pm 15,57 \\
53,81 \pm 15,62 \\
\end{array}$ & 0,057 & $\begin{array}{l}64,82 \pm 18,68 \\
61,04 \pm 18,11 \\
\end{array}$ & 0,112 & $\begin{array}{l}53,70 \pm 13,66) \\
49,57 \pm 13,41) \\
\end{array}$ & 0,018 & $\begin{array}{l}58,16 \pm 12,93) \\
54,95 \pm 11,87) \\
\end{array}$ & 0,046 & $\begin{array}{l}23,81 \pm 6,46) \\
23,43 \pm 6,99) \\
\end{array}$ & 0,663 \\
\hline
\end{tabular}


Research, Society and Development, v. 10, n. 7, e30810716626, 2021

(CC BY 4.0) | ISSN 2525-3409 | DOI: http://dx.doi.org/10.33448/rsd-v10i7.16626

(continuação)

\begin{tabular}{|c|c|c|c|c|c|c|c|c|c|c|c|c|}
\hline \multirow{2}{*}{ Variáveis } & \multicolumn{2}{|c|}{ Domínio Físico } & \multicolumn{2}{|c|}{ Domínio Psicológico } & \multicolumn{2}{|c|}{ Domínio Social } & \multicolumn{2}{|c|}{ Domínio Ambiental } & \multicolumn{2}{|c|}{ QV global } & \multicolumn{2}{|l|}{ PSS } \\
\hline & Média $\pm D P$ & $p$ & Média $\pm \mathrm{DP}$ & $p$ & Média \pm DP & $p$ & Média $\pm D P$ & $p$ & Média $\pm D P$ & $p$ & Média \pm DP & $p$ \\
\hline \multicolumn{13}{|l|}{ Tabagismo } \\
\hline $\begin{array}{l}\text { Sim } \\
\text { Não }\end{array}$ & $\begin{array}{l}60,72(22,19) \\
55,77(14,49)\end{array}$ & 0,382 & $\begin{array}{l}54,17(15,58) \\
55,86(15,71)\end{array}$ & 0,778 & $\begin{array}{l}66,68(18,62) \\
62,87(18,47)\end{array}$ & 0,591 & $\begin{array}{l}43,30(17,53) \\
51,92(13,51)\end{array}$ & 0,100 & $\begin{array}{l}56,21(15,42) \\
56,60(12,43)\end{array}$ & 0,935 & $\begin{array}{l}22,71(3,94) \\
23,66(6,78)\end{array}$ & 0,712 \\
\hline \multicolumn{13}{|l|}{ Etilismo } \\
\hline $\begin{array}{l}\text { Sim } \\
\text { Não }\end{array}$ & $\begin{array}{l}55,59(16,46) \\
55,92(13,93)\end{array}$ & 0,874 & $\begin{array}{l}55,85(15,37) \\
55,61(15,87)\end{array}$ & 0,912 & $\begin{array}{l}64,52(19,11) \\
62,27(18,21)\end{array}$ & 0,385 & $\begin{array}{l}51,19(14,39) \\
51,94(13,41)\end{array}$ & 0,695 & $\begin{array}{l}56,79(13,21) \\
56,43(12,24)\end{array}$ & 0,838 & $\begin{array}{l}24,41(6,62) \\
23,28(6,76)\end{array}$ & 0,227 \\
\hline \multicolumn{13}{|l|}{ Sono } \\
\hline $\begin{array}{l}\text { Sim } \\
\text { Não }\end{array}$ & $\begin{array}{l}61,41(15,77) \\
54,25(13,98)\end{array}$ & 0,001 & $\begin{array}{l}60,12(18,58) \\
54,47(14,47)\end{array}$ & 0,018 & $\begin{array}{l}66,21(17,86) \\
61,94(18,53)\end{array}$ & 0,128 & $\begin{array}{l}55,59(15,00) \\
50,49(13,02)\end{array}$ & 0,014 & $\begin{array}{l}60,83(14,11) \\
55,29(11,68)\end{array}$ & 0,003 & $\begin{array}{l}22,67(7,22) \\
23,91(6,53)\end{array}$ & 0,227 \\
\hline \multicolumn{13}{|l|}{ Atividade Social } \\
\hline $\begin{array}{l}\text { Sim } \\
\text { Não }\end{array}$ & $\begin{array}{l}59,15(15,23) \\
50,33(11,94)\end{array}$ & 0,000 & $\begin{array}{l}59,94(14,71) \\
48,82(14,86)\end{array}$ & 0,000 & $\begin{array}{l}67,50(17,00) \\
55,64(18,51)\end{array}$ & 0,000 & $\begin{array}{l}55,32(13,80) \\
46,72(11,84)\end{array}$ & 0,000 & $\begin{array}{l}60,47(12,18) \\
50,37(10,55)\end{array}$ & 0,000 & $\begin{array}{l}23,17(6,98) \\
24,42(6,43)\end{array}$ & 0,163 \\
\hline Refeição balance & & & & & & & & & & & & \\
\hline $\begin{array}{l}\text { Sim } \\
\text { Não }\end{array}$ & $\begin{array}{l}56,48(14,84) \\
52,12(13,27)\end{array}$ & 0,118 & $\begin{array}{l}56,35(15,54) \\
51,95(16,11)\end{array}$ & 0,136 & $\begin{array}{l}63,31(17,95) \\
60,42(21,47)\end{array}$ & 0,410 & $\begin{array}{l}52,39(13,51) \\
46,89(13,72)\end{array}$ & 0,033 & $\begin{array}{l}57,133(12,28) \\
52,841(13,26)\end{array}$ & 0,070 & $\begin{array}{l}23,56(6,77) \\
24,06(6,28)\end{array}$ & 0,696 \\
\hline Renda & & & & & & & & & & & & \\
\hline Sem renda & $54,46(14,43)$ & & $57,15(14,09)$ & & $70,83(14,34)$ & & $51,38(12,59)$ & & $58,45(10,33)$ & & $23,34(6,10)$ & \\
\hline Até $1 \mathrm{SM}$ & $54,40(12,89)$ & & $56,10(15,24)$ & & $60,07 \quad(19,19)$ & & $49,50(15,51)$ & & $55,02(13,28)$ & & $24,09(5,41)$ & \\
\hline 1 a $3 \mathrm{SM}$ & $54,41(13,91)$ & $0,623 *$ & $52,26(15,36)$ & $0,250^{*}$ & $57,51(18,09)$ & $<0,001 *$ & $48,78(12,10)$ & $0,019 *$ & $53,23(11,55)$ & $0,024 *$ & $24,24(6,24)$ & $0,908 *$ \\
\hline 3 a 6 SM & $57,62(17,12)$ & & $58,17(16,46)$ & & $69,87 \quad(14,30)$ & & $54,34(15,67)$ & & $59,99(13,14)$ & & $23,28(8,26)$ & \\
\hline Mais de $6 \mathrm{SM}$ & $57,82(13,89)$ & & $54,96(16,74)$ & & $59,93(24,50)$ & & $57,70(10,13)$ & & $57,60(13,08)$ & & $23,30(7,87)$ & \\
\hline
\end{tabular}

*ANOVA. Fonte: Autores. 
Em relação a qualidade do sono, alunos que referiram dormir de 7 a 8 horas diárias apresentaram melhores resultados nos domínios físico, psicológico e ambiental e QV global. Os graduandos que referiram ter atividades sociais regularmente apresentaram melhores resultados em todos os domínios da QV, e os que referiram realizar pelo menos uma refeição balanceada por dia tiveram melhor resultado no domínio ambiental (Tabela 2).

Os alunos que participavam de atividades de extensão apresentaram melhores resultados no domínio social, enquanto os que participavam de programas de pesquisa apresentaram melhores escores no domínio ambiental e QV global. Não houve diferenças significativas entre a realização de monitoria e escores de QV e estresse (Tabela 2).

$\mathrm{Na}$ comparação entre faixas de renda familiar mensal foi observado no pós-teste de Tukey que houve diferença significativa entre estudantes sem nenhuma renda e renda de um a três salários mínimos (SM) e desses com alunos de três a seis SM no domínio social. No domínio ambiental, houve diferença entre as rendas um a três SM e mais de seis salários. Na QV geral, houve diferença entre alunos com um a três salários e alunos com três a seis SM de renda familiar.

Na comparação entre os anos por campus, não houve diferenças significativas na QV no domínio físico e social. No entanto, observou-se melhores escores do domínio físico dos alunos do quinto ano em ambos os campi, enquanto no domínio social, alunos do terceiro ano apresentaram QV superior no campus ativo. Alunos do método ativo de ensino do primeiro e terceiro anos obtiveram QV superior no domínio psicológico. Os alunos do terceiro e quarto anos do método ativo obtiveram melhores escores nos domínios ambiental. A QV global dos alunos do terceiro ano do método ativo foi superior (Tabela 3). 
Tabela 3. Análise da qualidade de vida e estresse percebido segundo os métodos de ensino, por ano, sono e atividade social.

\begin{tabular}{|c|c|c|c|c|c|c|c|c|c|c|c|c|}
\hline & \multicolumn{2}{|l|}{$\begin{array}{c}\text { Domínio } \\
\text { Físico }\end{array}$} & \multicolumn{2}{|c|}{$\begin{array}{c}\text { Domínio } \\
\text { Psicológico }\end{array}$} & \multicolumn{2}{|c|}{$\begin{array}{c}\text { Domínio } \\
\text { Social }\end{array}$} & \multicolumn{2}{|c|}{$\begin{array}{c}\text { Domínio } \\
\text { Ambiental }\end{array}$} & \multicolumn{2}{|c|}{ QV global } & \multicolumn{2}{|c|}{ PSS } \\
\hline & Média $\pm D P$ & $p$ & Média $\pm D P$ & $p$ & Média $\pm \mathrm{DP}$ & $p$ & Média $\pm D P$ & $p$ & Média $\pm D P$ & $p$ & Média $\pm \mathrm{DP}$ & $p$ \\
\hline \multicolumn{13}{|l|}{$1^{\circ}$ ano } \\
\hline $\begin{array}{l}\text { Tradicional } \\
\text { Ativo }\end{array}$ & $\begin{array}{l}56,51 \pm 15,59 \\
55,98 \pm 12,94\end{array}$ & 0,895 & $\begin{array}{l}53,07 \pm 11,86 \\
60,56 \pm 12,89\end{array}$ & 0,037 & $\begin{array}{l}63,76 \pm 14,35 \\
66,66 \pm 21,03\end{array}$ & 0,576 & $\begin{array}{c}50,96 \pm 15,57 \\
55,81 \pm 9,90\end{array}$ & 0,183 & $\begin{array}{l}56,07 \pm 11,38 \\
59,75 \pm 10,13\end{array}$ & 0,228 & $\begin{array}{l}24,04 \pm 6,71 \\
22,82 \pm 6,31\end{array}$ & 0,507 \\
\hline \multicolumn{13}{|l|}{$2^{\circ}$ ano } \\
\hline $\begin{array}{l}\text { Tradicional } \\
\text { Ativo }\end{array}$ & $\begin{array}{l}51,23 \pm 15,05 \\
51,85 \pm 14,65\end{array}$ & 0,873 & $\begin{array}{l}53,13 \pm 14,54 \\
51,84 \pm 13,14\end{array}$ & 0,726 & $\begin{array}{l}56,78 \pm 19,21 \\
58,64 \pm 19,94\end{array}$ & 0,717 & $\begin{array}{l}47,57 \pm 14,19 \\
50,47 \pm 12,55\end{array}$ & 0,413 & $\begin{array}{l}52,18 \pm 12,17 \\
53,20 \pm 11,52\end{array}$ & 0,743 & $\begin{array}{l}23,84 \pm 7,38 \\
26,66 \pm 5,84\end{array}$ & 0,114 \\
\hline \multicolumn{13}{|l|}{$3^{\circ}$ ano } \\
\hline $\begin{array}{l}\text { Tradicional } \\
\text { Ativo }\end{array}$ & $\begin{array}{l}55,76 \pm 16,03 \\
60,55 \pm 12,91\end{array}$ & 0,266 & $\begin{array}{l}48,71 \pm 19,95 \\
64,01 \pm 14,75\end{array}$ & 0,005 & $\begin{array}{l}61,22 \pm 16,32 \\
71,58 \pm 20,52\end{array}$ & 0,057 & $\begin{array}{l}48,93 \pm 13,54 \\
57,67 \pm 15,78\end{array}$ & 0,045 & $\begin{array}{l}53,65 \pm 12,16 \\
63,45 \pm 13,78\end{array}$ & 0,012 & $\begin{array}{l}24,30 \pm 6,47 \\
20,81 \pm 7,07\end{array}$ & 0,081 \\
\hline \multicolumn{13}{|l|}{$4^{\circ}$ ano } \\
\hline $\begin{array}{l}\text { Tradicional } \\
\text { Ativo }\end{array}$ & $\begin{array}{l}55,16 \pm 13,03 \\
59,00 \pm 14,71\end{array}$ & 0,292 & $\begin{array}{l}53,94 \pm 16,00 \\
60,87 \pm 17,21\end{array}$ & 0,117 & $\begin{array}{l}59,86 \pm 16,42 \\
67,02 \pm 18,36\end{array}$ & 0,120 & $\begin{array}{l}47,96 \pm 10,80 \\
54,77 \pm 15,53\end{array}$ & 0,048 & $\begin{array}{l}54,23 \pm 10,83 \\
60,40 \pm 14,52\end{array}$ & 0,063 & $\begin{array}{l}23,18 \pm 6,43 \\
23,00 \pm 7,04\end{array}$ & 0,917 \\
\hline \multicolumn{13}{|l|}{$5^{\circ}$ ano } \\
\hline $\begin{array}{l}\text { Tradicional } \\
\text { Ativo }\end{array}$ & $\begin{array}{l}58,48 \pm 15,08 \\
63,38 \pm 20,54\end{array}$ & 0,513 & $\begin{array}{l}55,73 \pm 14,81 \\
65,11 \pm 13,15\end{array}$ & 0,144 & $\begin{array}{l}63,54 \pm 16,05 \\
69,78 \pm 17,77\end{array}$ & 0,395 & $\begin{array}{l}54,90 \pm 13,01 \\
54,31 \pm 15,66\end{array}$ & 0,923 & $\begin{array}{l}58,15 \pm 12,40 \\
63,13 \pm 15,10\end{array}$ & 0,397 & $\begin{array}{l}23,87 \pm 7,65 \\
23,12 \pm 4,67\end{array}$ & 0,803 \\
\hline \multicolumn{13}{|l|}{ Dorme $7 / 8 \mathrm{~h}$} \\
\hline $\begin{array}{l}\text { Tradicional } \\
\text { Ativo }\end{array}$ & $\begin{array}{l}59,76 \pm 17,08 \\
63,32 \pm 14,19\end{array}$ & 0,404 & $\begin{array}{l}57,78 \pm 20,43 \\
62,82 \pm 16,15\end{array}$ & 0,315 & $\begin{array}{l}58,33 \pm 16,80 \\
75,31 \pm 14,61\end{array}$ & 0,001 & $\begin{array}{l}50,11 \pm 14,52 \\
61,91 \pm 13,14\end{array}$ & 0,003 & $\begin{array}{l}56,50 \pm 14,61 \\
65,83 \pm 11,90\end{array}$ & 0,012 & $\begin{array}{l}23,30 \pm 6,67 \\
21,96 \pm 7,89\end{array}$ & 0,495 \\
\hline \multicolumn{13}{|l|}{ Atividade social } \\
\hline $\begin{array}{l}\text { Tradicional } \\
\text { Ativo }\end{array}$ & $\begin{array}{l}57,87 \pm 14,92 \\
60,77 \pm 15,59 \\
\end{array}$ & 0,265 & $\begin{array}{l}56,41 \pm 14,61 \\
64,38 \pm 13,69 \\
\end{array}$ & 0,001 & $\begin{array}{l}63,78 \pm 15,87 \\
72,17 \pm 17,33\end{array}$ & 0,004 & $\begin{array}{l}52,73 \pm 13,86 \\
58,58 \pm 13,13 \\
\end{array}$ & 0,012 & $\begin{array}{l}57,70 \pm 11,60 \\
63,96 \pm 12,09 \\
\end{array}$ & 0,002 & $\begin{array}{l}24,12 \pm 7,05 \\
21,96 \pm 6,76\end{array}$ & 0,069 \\
\hline
\end{tabular}

Fonte: Autores. 
Alunos do método ativo que dormiam mais de 7/8 horas apresentaram melhor QV nos domínios social e ambiental e QV global, enquanto aqueles que realizavam atividades sociais regularmente no mesmo campus obtiveram melhores resultados nos domínios psicológico, social e ambiental e QV global.

Não houve diferença significativa entre os resultados dos alunos que fazem monitoria e extensão em relação ao campus. Os alunos que fazem pesquisa no método ativo de ensino apresentaram melhor resultado no domínio psicológico $(61,186 ; p<0,018)$. Não houve nenhuma diferença significativa entre a percepção de estresse entre os alunos do método tradicional e ativo de ensino.

\section{Discussão}

O método ativo influenciou positivamente na percepção da QV em comparação aos alunos do método tradicional de ensino em diferentes aspectos, porém não houve associação significativa entre a abordagem pedagógica e a percepção do estresse dos graduandos de enfermagem.

Os testes de associação também não mostraram diferenças significativas entre as variáveis sociodemográficas, acadêmicas e hábitos de vida e o estresse. No entanto, observou-se que houve uma correlação negativa entre o estresse e todos os domínios da QV e da QV geral. Em um estudo realizado com acadêmicos da área da saúde, observou-se a mesma correlação, de modo que quanto maior a percepção de estresse, menor a QV dos estudantes (Bornie, Wanderley \& Bassitt, 2015).

Algumas variáveis foram relacionadas aos desfechos de QV. Na comparação entre os métodos de ensino, alunos do campus ativo apresentaram maiores escores de QV no domínio social. Atribui-se a isso a valorização e estímulo à interação e o desenvolvimento de habilidades de comunicação, negociação, autonomia e responsabilidade nessa abordagem pedagógica. O método de aprendizagem baseado em problemas estimula a construção coletiva do conhecimento, enfatiza a crítica, a discussão e a reflexão sobre os aspectos sociais e as relações que envolvem o cuidado em enfermagem (Santos, Nunes, Demarco \& Amestoy, 2017).

A realização de atividades sociais regulares foi associada a uma melhor percepção de QV em todos os domínios. Entre os alunos da metodologia PBL, esse resultado foi superior nos domínios psicológico, social e ambiental e QV global. Isso pode estar relacionado ao fato de os alunos do campus ativo residir, predominantemente, em repúblicas com amigos, localizadas no mesmo município da universidade. O suporte social de amigos e parentes foi relacionado às maiores pontuações no domínio social em um estudo realizado na região sul do Brasil. Além disso, a localização da universidade longe de casa pode ser fator que contribui para capacitar os alunos em suas relações sociais (Moritz et al., 2016).

Estudantes que referiram nenhuma renda familiar apresentaram melhor QV no domínio social que aqueles com renda de um a três salários mínimos. Isso também pode ser explicado pelo fato de os alunos do método ativo residirem predominantemente em repúblicas e, por isso, não terem considerado a renda da família. Assim como nesse estudo, existe um perfil de acadêmicos de enfermagem que geralmente não trabalham, em contrapartida, a baixa renda familiar pode exigir que os estudantes procurem empregos para sustentar suas necessidades financeiras (Cruz et al., 2018).

Estudantes que trabalhavam apresentaram menores escores no domínio social. O curso de enfermagem é ofertado, em ambos os campi, de forma integral, o que dificulta ou impede que o aluno disponha de tempo livre para exercer atividades remuneradas. Aqueles que as exercem podem apresentar maiores limitações nas suas relações sociais extraclasse e na realização de atividades recreativas. A falta de tempo para estar com os amigos e realizar atividades de lazer foi referida como a maior causa de estresse universitário (Hirsch et al., 2018).

O tempo consumido no deslocamento e o uso do transporte público foram identificados como fatores de desgaste para os estudantes de enfermagem (Hirsch et al., 2018). O campus ativo está localizado em um município do interior do estado, 
onde as distâncias percorridas entre a moradia, a universidade e os campos de estágios são encurtados. Dessa forma, o aluno pode gerenciar melhor o tempo e conciliar melhor as atividades acadêmicas ao convívio social.

Os graduandos com renda familiar superior a seis salários mínimos apresentaram melhor desempenho no domínio ambiental. Considerando-se que esse domínio está relacionado ao acesso a segurança física, proteção, ambiente físico e do lar, recursos financeiros, transporte, oportunidade de lazer e de adquirir novos conhecimentos e habilidades (Fleck et al., 1999), infere-se que a renda familiar pode ser preditora de uma melhor QV, como revelado por um estudo multinacional (Cruz et al., 2018).

Não houve diferença significativa entre a percepção de estresse dos graduandos nos diferentes métodos de aprendizagem. Porém, os resultados indicam uma correlação significativa moderada entre o nível de estresse e o domínio psicológico, fato este também observado em outro estudo (Oliveira et al., 2015). O estresse é considerado uma experiência em que as demandas de uma situação física ou psicológica excedem a capacidade percebida de enfrentá-la. Desse modo, o estressor afeta o organismo humano de modo diverso, sobrecarregando-o ou excedendo seus recursos adaptativos (Nodari et al., 2014). A presença de sentimentos negativos, mau humor, ansiedade e desespero foi relacionada ao menor escore no domínio psicológico (Moritz et al., 2016).

O domínio psicológico também está relacionado a capacidade de aprendizagem, memória e concentração (WHO, 1997). Neste estudo, a QV no domínio psicológico dos alunos do método ativo foi superior. Estudo realizado no Irã revelou que acadêmicos de enfermagem submetidos à PBL apresentaram melhores níveis de desempenho geral durante as atividades de aprendizagem, com um menor esforço mental que os alunos do método convencional. Além disso, o método ativo foi relacionado ao aumento da carga cognitiva dos alunos e a retenção do conhecimento a longo prazo (Salari et al., 2018).

De maneira inesperada, alunos do primeiro ano da graduação do método ativo apresentaram uma melhor percepção da QV no domínio psicológico. Em virtude do desafio de adaptar-se a um novo modo de aprender, após uma vida escolar baseada no método tradicional, esperava-se que esses alunos tivessem uma redução na percepção da QV. Estudo comparativo revelou que alunos submetidos à metodologia PBL apresentaram maiores escores de estresse que os alunos da metodologia baseada em palestras nesse período (Wardley, Applegate, Almaleki \& Van Rhee, 2016).

Esse resultado pode estar relacionado a alta carga horária ofertada no método tradicional nesse ano (primeiro e segundo semestres), concentrada em disciplinas teóricas complexas das ciências da saúde. Nessa fase, os alunos têm pouco contato com disciplinas específicas da enfermagem e podem apresentar dificuldade em correlacionar o conhecimento adquirido com a futura profissão, o que pode contribuir para frustação das expectativas. Além disso, o método tradicional de aprendizagem pode desencorajar os alunos, devido a troca passiva de conhecimentos (Magnavita \& Chiorri, 2018).

Neste estudo, no terceiro ano, alunos do método convencional de ensino apresentaram menor percepção na QV global e nos domínios psicológico e ambiental. A diminuição da satisfação nesse período pode ser atribuída a inserção dos alunos em disciplinas específicas que exigem o desenvolvimento de habilidades e competências teórico-práticas e a assistência de enfermagem em ambiente hospitalar, como observado em um estudo realizado em uma Instituição de Ensino Superior paulista. De forma equivalente, o referido estudo observou maiores índices de insatisfação entre alunos do segundo ano (Bampi et al., 2013).

Corroborando com outros estudos (Bampi et al., 2013; Oliveira et al., 2015), o domínio ambiental teve a pior média em ambos os campi. Apesar de também estar inseridos em ambiente hospitalar, os alunos de metodologia ativa do terceiro e quarto anos tiveram uma percepção melhor nessa faceta. Isso pode estar relacionado ao ensino de técnicas e habilidades em laboratórios de simulação nesse método. Devido ao ambiente clínico simulado, procura-se fornecer um espaço de aprendizagem positivo, com estímulo ao pensamento crítico para resolução de problemas e o trabalho em equipe, preparando o acadêmico para a prática clínica (Jeppesen, Christiansen \& Frederiksen, 2017). 
No método convencional, os alunos desempenham um papel mais passivo (Salari et al., 2016), no qual instruções são ditadas para realização da prática em circunstâncias favoráveis. Um estudo espanhol identificou entre as situações mais estressantes vivenciadas por estudantes de enfermagem a prática clínica e o medo de causar danos aos pacientes ou a si mesmos. Isso pode estar relacionado à falta de tempo, falta de material ou falha na implementação de técnicas sob as "condições ideais" ensinadas nas aulas teóricas convencionais (Suárez-García et al., 2018).

No último ano da graduação, os alunos de ambos os campi estavam inseridos no estágio supervisionado, período em que assumem o papel de enfermeiros no seu ambiente de trabalho sob supervisão. Isso pode explicar a ausência de diferença significativa na comparação da QV e percepção de estresse nessa série.

Associadas ao ensino, as práticas pedagógicas de pesquisa e extensão completam o tripé da formação superior. Neste estudo, essas atividades foram associadas a melhor QV dos graduandos em enfermagem. Além de atuar no reconhecimento das necessidades sociais e tornar-se consciente da sua realidade (Oliveira et al., 2015), alunos que participavam de projeto de extensão apresentaram QV superior no domínio social.

Alunos que participavam de projetos de iniciação científica apresentaram melhor QV global e no domínio ambiental. A pesquisa na graduação em enfermagem propicia o desenvolvimento do pensamento crítico, aproxima o aluno da realidade social e contribui para o amadurecimento profissional, apesar disso, estudos mostram que o incentivo das instituições de ensino a inserção e preparação dos alunos para essa prática ainda é deficitária (Araújo et al., 2015; Santos, Anjos \& Almeida, 2015).

Assim como em outros estudos (Araújo et al., 2013; Santos et al., 2016), grande parte dos participantes desta pesquisa referiram dormir menos de sete a oito horas por dia, o que foi associado a menor QV nos domínios físico, psicológico, ambiental e QV geral. Quando o sono não viabiliza o descanso físico e mental efetivo, há comprometimento da capacidade de concentração do graduando, visto que implica na redução da energia para executar atividades diárias (Pereira, Pinho \& Cortes, 2013). Além disso, estudo revelou que a má qualidade do sono em estudantes de enfermagem estava intimamente associada com sintomas depressivos (Zhang, Peters \& Bradstreet, 2017).

Não houve influência da abordagem pedagógica no domínio físico. Porém, entre os que tinham uma média de horas de sono maior que sete, graduandos do método tradicional obtiveram menor QV nos domínios social, ambiental e QV global. Foi observado que aspectos relacionados a formação profissional, como conflitos presentes nas relações com colegas e profissionais de saúde, as preocupações do discente com a aquisição de conhecimento, inserção no mercado de trabalho e a dificuldade em relação aos conteúdos apreendidos e às metodologias de ensino, impactou na qualidade do sono dos estudantes (Benavente et al., 2014).

Algumas limitações metodológicas deste estudo devem ser consideradas. A amostra obtida por conveniência e a inclusão de apenas uma abordagem metodológica ativa pode limitar a generalização dos resultados. Além disso, a indisponibilidade de tempo dos alunos para responder aos instrumentos foi um obstáculo durante a coleta de dados. Partindo do pressuposto que os níveis de estresse variam ao longo do tempo e dependem de fatores da vida diária (Lazarus \& Folkman, 1987), refletindo também na sua percepção de QV, como visto neste estudo, a abordagem longitudinal poderia detectar melhor as mudanças nos níveis de estresse dos acadêmicos e percepção da QV ao decorrer da graduação.

Apesar disso, os resultados deste estudo demonstram a importância da comparação da QV e estresse entre graduandos de enfermagem de métodos de aprendizagem tradicional e ativa. Naturalmente, durante a passagem pela universidade, diversos fatores podem contribuir para redução da QV e maior percepção de estresse, como o excesso de atividades acadêmicas, o deslocamento até a faculdade e aos locais de estágio, e a falta de tempo para estar com a família, parentes ou ainda para realizar atividades de lazer (Hirsch et al., 2018). Porém, observou-se que características do próprio método de ensino podem ser determinantes na redução ou melhor percepção da QV entre os acadêmicos. 
Atenção especial deve ser dada aos aspectos psicológicos influenciados pelo processo de formação em enfermagem, visto que foi observado níveis equivalentes de estresse entre os alunos, apesar da abordagem metodológica distinta. Sugere-se ainda que estudos posteriores possam avaliar se o tempo protegido para estudo autodirigido, presente no método ativo, possa ser considerado um fator protetivo para QV.

Dessa forma, espera-se que alguns aspectos metodológicos possam ser revistos pelos docentes e instituições de ensino, a fim de proporcionar um aprendizado mais ativo e motivador, melhorando assim o desempenho dos alunos e a sua QV. Além disso, estratégias de apoio e enfrentamento aos estressores, baseadas nas reais necessidades desses estudantes, devem ser adotadas.

\section{Conclusão}

Graduandos do método ativo de ensino-aprendizagem apresentaram QV superior nos domínios psicológico, social, ambiental e QV global. Na PBL, alunos do primeiro ano e terceiro ano apresentaram desempenho superior no domínio psicológico, assim como alunos do terceiro e quarto anos obtiveram melhor média no domínio ambiental. Dormir de sete a oito horas diárias proporcionou maior QV dos acadêmicos do campus ativo nos domínios social, ambiental e QV global. Assim como apresentaram melhor QV nos domínios psicológico, social, ambiental e QV global, entre os que realizavam atividades social regularmente. Não houve diferença estatística significante entre os alunos do campus ativo e tradicional a respeito da percepção de estresse. Porém houve correlação negativa significativa entre os domínios do WHOQOL-bref e a PSS.

\section{Referências}

Araújo, A., Morais, H., Vasconcelos, H., Rabelo, J. C., Santos, R. X. L., \& Holanda, R. E. (2015). A pesquisa científica na graduação em enfermagem e sua importância na formação profissional. Rev enferm UFPE online, 9(9), 9180-9187.

Araújo, M., Lima, A., Alencar, A., Araújo, T., Fragoaso, L., \& Damasceno, M. (2013). Avaliação da qualidade do sono de estudantes universitários de Fortaleza-CE. Texto Context Enferm, 22(2), 352-360.

Bampi, L. N. S., Caraldi, S., Guilhem, D., Pompeu, R. B., \& Campos, A. C. O. (2013). Percepção sobre qualidade de vida de estudantes de graduação em enfermagem. Rev Gaúcha Enferm, 34(1), 125-132.

Benavente, S. B. T., Silva, R. M., Higashi, A. B., Guido, L. A., \& Costa, A. L. S. (2014). Influência de fatores de estresse e características sociodemográficas na qualidade do sono de estudantes de enfermagem. Rev da Esc Enferm, 48(3), 514-520.

Bornie, R. C. C., Wanderley, K. S., \& Bassitt, D. P. (2015). Relação entre a qualidade de vida e o estresse em acadêmicos da área da saúde. Estud Interdiscip Psicol, 6(1), 100-118.

Cohen, S. \& Williamson, G. (1988). Perceived stress in a probability sample of the United States. The Social Psychology of Health.

Cohen, S., Kamarck, T., \& Mermelstein, R. (1983). A global measure of perceived stress. J Health Soc Behav, 24(4), $385-396$

Cruz, J. P., Felicilda-Reynaldo, R. F. D., Lam, S. C., Contreras, F. A. M., Cecily, H. S. J., Papathanasiou, I. V., et al. (2018). Quality of life of nursing students from nine countries : A cross-sectional study. Nurse Educ Today, 66, 135-142.

Fleck, M. P. D. A., Leal, O. F., Louzada, S., Xavier, M., Chachamovich, E., Vieira, G., et al. (1999). Desenvolvimento da versão em português do instrumento de avaliação de qualidade de vida da OMS: WHOQOL-100. Rev Bras Psiquiatr, 21(1),19-28.

Gouveia, M. T. D. O., Santana, H. S., Maria, A., Ribeiro, G., \& Fernandes, M. A. (2017). Quality of life and welfare of nursing university students: integrative review. REUFPI, 6(3), 72-78.

Hirsch, C. D., Luiz, E., Barlem, D., \& Almeida, L. K. (2018). Stress triggers in the educational environment from the perspective of nursing students. Texto contexto enferm, 27(1), e0370014.

Jeppesen, K. H., Christiansen, S., \& Frederiksen K. (2017). Education of student nurses: a systematic literature review. Nurse Educ Today, 55,112-121.

Lazarus, R. S. \& Folkman, S. (1987). Transactional theory and research on emotions and coping. Eur J Pers, 1(3), $141-169$.

Magnavita, N. \& Chiorri, C. (2018). Academic stress and active learning of nursing students: A cross-sectional. Nurse Educ Today, 68, 128-133.

Moritz, A. R., Pereira, E. M., Borba, K. P., Clapis, M. J., Gevert, V. G., \& Mantovani, M. F. (2016). Quality of life of undergraduate nursing students at a Brazilian public university. Investig Educ Enfermería, 34(3), 564-572. 
Research, Society and Development, v. 10, n. 7, e30810716626, 2021

(CC BY 4.0) | ISSN 2525-3409 | DOI: http://dx.doi.org/10.33448/rsd-v10i7.16626

Nodari, N. L., Flor, S. R. A., Ribeiro, A. S., Hayasida, N. M. A., \& Carvalho, G. J. R. (2014). Estresse, conceitos, manifestações e avaliação em saúde: revisão de literatura. Rev Saúde Desenvolv Hum, 2(1), 61-74.

Oliveira, F. L. B. \& Almeida Júnior, J. J. (2015). Extensão universitária: contribuições na formação de discentes de Enfermagem. Rev Bras Pesq Saúde, 17(1), 19-24.

Oliveira, H. F. R., Risso, H. R. F., Vieira, F. S. F., Leal, K. A. S., Novelli, C., Noda, D. K. G., et al. (2015). Stress and quality of life of University students. Rev CPAQV, 7(2), 1-8.

Pereira, M. O., Pinho, P. H., \& Cortes, J. M. (2016). Qualidade de vida: percepção de discentes de graduação em enfermagem. J Nurs Heal, 6(2), 321 -333.

Pinto, A. A. M., Marin, M. J. S., Tonhom, S. F. R., \& Ferreira M. L. S. M. (2016). Métodos de ensino na graduação em enfermagem: uma revisão integrativa da literatura. Atas CIAIQ, 1, 971-980.

Quadro, J. S. \& Colomé, J. S. (2016). Metodologias de ensino-aprendizagem na formação do enfermeiro. Rev Baiana Enferm, 30(2), 1-10.

Salari, M., Roozbehi, A., Zarifi, A., \& Tarmizi, R. A. (2018). Pure PBL, Hybrid PBL and Lecturing: which one is more effective in developing cognitive skills of undergraduate students in pediatric nursing course? BMC Med Educ, 18(195), 1-15.

Santos, E. O., Nunes, C. K., Demarco, D. A., \& Amestoy, S. C. (2017). Aprendizagem baseada em problemas no ensino da enfermagem. Rev Context Saúde, 17(32), 55-66.

Santos, T., Martino, M., Sonati, J., Faria, A., \& Nascimento, E. (2016). Qualidade do sono e cronotipo de estudantes de enfermagem. Acta Paul Enferm, 29(6), 658-663.

Santos, V. C., Anjos, K. F., \& Almeida, O. S. (2015). Iniciação científica a partir de estudantes de Enfermagem. Rev Bras Cien Saúde, 2015;19(4):255-60.

Selye, H. (1951). The Physiology and Pathology of Exposure to Stress. Science, 113(2938), 462-463.

Suárez-García, J. M., Maestro-González, A., Zuazua-Rico, D., Sánchez-Zaballos, M., \& Mosteiro-Diaz, M. P. (2018). Stressors for spanish nursing students in clinical practice. Nurse Educ Today, 64, 16-20.

Wardley, C. S., Applegate, E. B., Almaleki, A. D., \& Van Rhee, J. A. (2016). A comparison of students' perceptions of stress in parallel problem-based and lecture-based curricula. J Physician Assist Educ, 27(1), 7-16.

World Health Organization. (1997). WHOQOL: measuring quality of life. Psychol Med, 28(3), 551-558.

World Health Organization. (2009). Global standards for the initial education of professional nurses and midwives. https://www.who.int/hrh/nursing_midwifery/hrh_global_standards_education.pdf

Zhang, Y., Peters, A., \& Bradstreet, J. (2017). Relationships among sleep quality, coping styles, and depressive symptoms among college nursing students: A multiple mediator model. J Prof Nurs, 34(4), 320-325. 